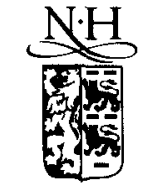

ELSEVIER

\title{
An integrable model of quantum gravity
}

\author{
D. Korotkin ${ }^{\mathrm{a}, \mathrm{b}, 1}, \mathrm{H}$. Nicolai ${ }^{\mathrm{a}}$ \\ a II. Institute for Theoretical Physics, Hamburg University, Luruper Chaussee 149, 22761 Hamburg, Germany \\ b Steklov Mathematical Institute, Fontanka, 27, St. Petersburg 191011, Russia \\ Received 26 May 1995; revised manuscript received 27 June 1995 \\ Editor: P.V. Landshoff
}

\begin{abstract}
We present a new quantization scheme for $2 \mathrm{D}$ gravity coupled to an SU(2) principal chiral field and a dilaton; this model represents a slightly simplified version of stationary axisymmetric quantum gravity. The analysis makes use of the separation of variables found in our previous work [D. Korotkin and H. Nicolai, Phys. Rev. Lett. 74 (1995) 1272] and is based on a two-time Hamiltonian approach. The quantum constraints are shown to reduce to a pair of compatible first order equations, with the dilaton playing the role of a "clock field". Exact solutions of the Wheeler-DeWitt equation are constructed via the integral formula for solutions of the Knizhnik-Zamolodchikov equations.
\end{abstract}

\section{Introduction}

At present only few models of quantum gravity exist for which the Wheeler-DeWitt equation can be solved exactly. The known examples include pure gravity [2] and supergravity [3] in three dimensions, as well as certain mini-superspace models such as static spherically symmetric gravity [4] or locally supersymmetric Bianchi-type models [5]. All of these models describe only finitely many physical degrees of freedom and are thus bound to miss essential features of quantum gravity. A simple example of a model with infinitely many physical degrees of freedom - the quantum theory of cylindrical gravitational fields - was studied in [6]. In this paper we present a model of quantum gravity describing 2D gravity coupled to a principal chiral SU(2) model and a dilaton, and demonstrate that it can be quantized exactly by use of methods developed in the context of $2 \mathrm{D}$ integrable systems $[7,8]$. The construction is based on our previous work [1], where we have proposed a novel canonical formulation of the Ernst equation based on the complete separation of the equations of motion in the isomonodromic sector of the theory and the use of the logarithmic derivative of the related $\Psi$ function with respect to the spectral parameter as the fundamental canonical variable. Our model can be regarded as a version of the "midi-superspace" approximation of quantum gravity, but differs from dimensionally reduced gravity in that we have replaced the non-compact configuration space $\mathrm{SU}(1,1) / \mathrm{U}(1)$ by the compact group manifold SU(2). Apart from this technical modification (the reasons for which will be briefly explained at the end of this paper) the model captures all the essential features of dimensionally reduced quantum gravity, and is a non-trivial example of a completely integrable model of quantum gravity with infinitely many propagating degrees of freedom. A further intriguing aspect of the present work is the possible relevance of quantum groups suggested by the link with the Knizhnik-Zamolodchikov (KZ) equations.

\footnotetext{
${ }^{1}$ Supported by Alexander von Humboldt Foundation.
} 


\section{Classical model}

For definiteness we consider only Euclidean worldshcets with complex local coordinates $(\xi, \bar{\xi})$ (the extension of our results to Lorentzian worldsheets is completely straightforward). The worldsheet metric is

$d s^{2}=h(\xi, \bar{\xi}) d \xi d \bar{\xi}, \quad h \equiv e^{2 k}$

and the Lagrangian is taken to be

$\mathcal{L}=\rho\left(R+\operatorname{tr}\left(g_{\xi} g^{1} g_{\bar{\xi}} g^{-1}\right)\right)$.

Here $\rho(\xi, \bar{\xi}) \in \mathbb{R}$ is the dilaton ficld originating from dimensional reduction as explained, e.g. in [9], and the chiral field $g(\xi, \bar{\xi}) \in G=S U(2)$ describes the matter content of the model (subscripts stand for partial derivatives). The conformal factor in (1) represents a further, but dependent, degree of freedom and appears in (2) via the Gaussian curvature of the worldsheet; in the conformal gauge this term becomes $R=$ $(\log h)_{\xi \xi} / h$. The equation of motion for $\rho(\xi, \bar{\xi})$ following from (2) reads

$\rho_{\xi \bar{\xi}}=0$

and possesses the general solution $\rho(\xi, \bar{\xi})=f(\xi)+$ $f(\bar{\xi})$. Assuming $f \neq$ const. we can perform an analytic local change of coordinates such that $f(\xi)=$ $\xi / 2 i$ (global aspects of this change of variables were discussed in [10], but will not concern us here). In the following we shall thus always assume

$\rho(\xi, \bar{\xi})=\operatorname{Im} \xi$

and also set $x(\xi, \bar{\xi}):=\operatorname{Re} \xi$. We will take some liberty in referring to $\rho$ and $x$ as the "time" and "space" coordinates, respectively. The gauge choice (4) is analogous to the light cone gauge in string theory whereby one identifies the longitudinal target space coordinate $X^{+}$with the world sheet time coordinate. The remaining (and compatible) equations of motion read

$2 k_{\xi}=\frac{\xi-\bar{\xi}}{4} \operatorname{tr}\left(g_{\xi} g^{-1}\right)^{2}$,

$2 k_{\bar{\xi}}=\frac{\bar{\xi}-\xi}{4} \operatorname{tr}\left(g_{\bar{\xi}} g^{-1}\right)^{2}$

and

$$
\left((\xi-\bar{\xi}) g_{\xi} g^{-1}\right)_{\bar{\xi}}+\left((\xi-\bar{\xi}) g_{\bar{\xi}} g^{-1}\right)_{\xi}=0 \text {. }
$$

As is evident from these equations, the $2 \mathrm{D}$ gravitational fields and the matter fields are coupled through the dilaton $\rho$; furthermore it can be shown that the obvious solution $\rho=$ const. of (3) would imply $g_{\xi}=$ $g_{\bar{\xi}}=h_{\xi}=h_{\bar{\xi}}=0$, i.e. the trivial solution for the matter fields, in which case the gravitational sector would become purely topological ( strictly speaking, this argument relies on the positive definiteness of the Cartan Killing metric on the group $G$ ). Therefore the model possesses no non-trivial flat space limit since the matter fields act as sources for 2D gravity and thus distort the two-dimensional background geometry.

Eq. (6) is the compatibility condition of the following linear system [11]:

$$
\begin{aligned}
& \frac{\partial \Psi}{\partial \xi}+\frac{\gamma}{\xi-\bar{\xi}} \frac{1+\gamma}{1-\gamma} \frac{\partial \Psi}{\partial \gamma}=\frac{g_{\xi} g^{-1}}{1-\gamma} \Psi, \\
& \frac{\partial \Psi}{\partial \bar{\xi}}+\frac{\gamma}{\bar{\xi}-\xi} \frac{1-\gamma}{1+\gamma} \frac{\partial \Psi}{\partial \gamma}=\frac{g_{\bar{\xi}} g^{-1}}{1+\gamma} \Psi
\end{aligned}
$$

where $\Psi \equiv \Psi(\xi, \bar{\xi} ; \gamma)$ is a two-by-two matrix, from which the solution of (6) can be reconstructed, and $\gamma$ is a "variable spectral parameter",

$$
\gamma(\xi, \bar{\xi} ; w)=\frac{2}{\xi-\bar{\xi}}\left\{w-\frac{\xi+\bar{\xi}}{2}+\sqrt{(w-\xi)(w-\bar{\xi})}\right\}
$$

with $w \in \mathbb{C}$ a constant of integration which may be regarded as the "hidden" spectral parameter. Notice that the poles in the $\gamma$ plane appearing on the 1.h.s. of (7) are produced by differentiation of $\gamma$ with respect to $\xi$ and $\bar{\xi}$, respectively.

According to the approach developed in [1], the fundamental quantities are the logarithmic derivatives of $\Psi$ with respect to the spectral parameter $\gamma$ :

$$
\Psi_{\gamma}=A(\gamma) \Psi, \quad A(\gamma) \equiv \sum_{j} \frac{A_{j}}{\gamma-\gamma_{j}}
$$

where for notational clarity we have suppressed the dependence on the coordinates $(\xi, \bar{\xi})$. The two-by-two matrices $A_{j}(\xi, \bar{\xi})$ are the residues at $\gamma_{j}=\gamma\left(\xi, \bar{\xi} ; w_{j}\right)$ in the complex $\gamma$-plane, with constants $w_{j}$. We here consider only the isomonodromic sector, for which the sum (9) is finite; in general, however, the sum (9) may contain an infinite or even continuous number of terms (if the sum is replaced by an integral). 
The compatibility conditions of (9) and (7) yield the following system of differential equations for the matrices $A_{j}(\xi, \bar{\xi})[1]$ :

$$
\begin{aligned}
& A_{j \xi}=\frac{2}{\xi-\bar{\xi}} \sum_{k \neq j} \frac{\left[A_{k}, A_{j}\right]}{\left(1-\gamma_{k}\right)\left(1-\gamma_{j}\right)}, \\
& A_{i \bar{\xi}}=\frac{2}{\bar{\xi}-\xi} \sum_{k \neq j} \frac{\left[A_{k}, A_{j}\right]}{\left(1+\gamma_{k}\right)\left(1+\gamma_{j}\right)} .
\end{aligned}
$$

As emphasized in $[1]$ these equations are automatically compatible unlike the original linear system (7). The problem of solving the nonlinear partial differential equation (6) is thus reduced to solving a system of ordinary matrix differential equations. Once the solutions $A_{j}$ of (10) are known, the chiral field $g(\xi, \bar{\xi})$ can be recovered from $A(\gamma)$ by specializing the spectral parameter $\gamma$ to the values $\gamma= \pm 1$, viz.

$$
\begin{aligned}
& (\xi-\bar{\xi}) g_{\xi} g^{-1}=\left.2 A(\gamma)\right|_{\gamma=1}, \\
& (\xi-\bar{\xi}) g_{\bar{\xi}} g^{-1}=\left.2 A(\gamma)\right|_{\gamma=-1} ;
\end{aligned}
$$

again we have not displayed the dependence on the coordinates in these expressions. The conformal factor $h(\xi, \bar{\xi})$ may then be computed by integration of $(5)$. In [1] we have shown that it is essentially the $\tau$ function associated with (7) in the sense of [12].

All results so far are actually valid for arbitrary $\operatorname{SL}(2, \mathbb{C})$ (and, in fact, $\operatorname{GL}(n, \mathbb{C})$ ) valued matrices $g(\xi, \bar{\xi})$, but we will consider only $G=\mathrm{SU}(2)$ in the remainder of this paper. The solutions of the system (10) corresponding to $g \in \mathrm{SU}(2)$ and $h \in \mathbb{R}$ are characterized by the following

Lemma. Let $\left\{A_{j}, t_{j} ; j=1, \ldots, N\right\}$ be invariant with respect to the involution $A_{j} \rightarrow-A_{j}^{\dagger}, \gamma_{j} \rightarrow-\bar{\gamma}_{j}$ (i.e. if $\gamma_{j}=-\bar{\gamma}_{j}$ then $A_{j} \in \operatorname{su}(2)$; if $\gamma_{j}=-\bar{\gamma}_{k}, k \neq j$ then $A_{j}=-A_{k}^{\dagger}$ ). Then the constants of integration in (5) and (11) may be chosen in such a way that $g \in \mathrm{SU}(2)$ and $h \in \mathbb{R}$.

The conditions of Lemma 1 in particular imply that $A(1)^{\dagger}=A(-1)$. In the following we restrict our attention to the case $\gamma_{j}=-\overline{\gamma_{j}}$ (i.e. $w_{j} \in \mathbb{R}$ ), so that $A_{j} \in \operatorname{su}(2)$ for all $j$. Regularity of $\Psi(\gamma)$ at $\gamma=\infty$ together with (9) implies a further constraint, viz.

$\sum_{j=1}^{N} A_{j}=0$.
This condition is related to the asymptotical flatness of the corresponding classical solutions. All constraints are consistent with the equations of motion (10) and form a closed algebra with respect to the Poisson bracket (13) below.

It was also explained in [1] that Eqs. (10) can he interpreted as a two-time Hamiltonian system with respect to "times" $\xi$ and $\bar{\xi}$. The relevant Poisson brackets are given by ${ }^{2}$

$$
\{A(\gamma) \stackrel{\otimes}{A} A(\mu)\}=[r(\gamma-\mu), A(\gamma) \otimes I+I \otimes A(\mu)]
$$

where the classical $r$-matrix $r(\gamma)$ is equal to $\Pi / \gamma$ with II the permutation operator in $\mathbb{C}^{2} \times \mathbb{C}^{2}$. The dynamics in the $\xi$-and $\bar{\xi}$-directions is governed by the complex Hamiltonians

$$
H^{(\xi)}=\frac{1}{\xi-\bar{\xi}} \operatorname{tr}\left(A^{2}(1)\right),
$$

and

$$
H^{(\bar{\xi})}=\frac{1}{\bar{\xi}-\xi} \operatorname{tr}\left(A^{2}(-1)\right)
$$

respectively, which depend explicitly on $(x, \rho)$ through the prefactor $\rho^{-1}$ and the spectral values $\gamma_{j}$ via (8). Observe that we have $\left(H^{(\xi)}\right)^{\dagger}=H^{(\bar{\xi})}$ by Lemma 1. As expected from the decoupling of variables in $(10)$, the flows generated by $H^{(\xi)}$ and and $H^{(\bar{\xi})}$ commute, i.e. $\left\{H^{(\xi)}, H^{(\bar{\xi})}\right\}=0$, and this ensures that the equations of motion can be consistently integrated. Remarkably, the dimension of the system has been effectively reduced by trading the "space" variable $x$ and the "time" variable $\rho$ for two "time" variables $\xi$ and $\bar{\xi}$. We can thus neglect the dependence on $x$ and $\rho$ for all practical purposes and regard the spectral parameter currents $A(\gamma)$ at a fixed but arbitrarily chosen base point $\left(x_{0}, \rho_{0}\right)$ as the fundamental canonical variables.

\footnotetext{
${ }^{2}$ For readers not familiar with the combined tensor product and Poisson bracket notation of [7], we spell out this equation once more with matrix indices $a, b, \ldots$ (which here assume only two values) written out explicitly: $\left\{A_{a b}(\gamma), A_{c d}(\mu)\right\}=$

$\frac{1}{\gamma-\mu}\left(\delta_{a d}(A(\gamma)-A(\mu))_{c b}-\delta_{c b}(A(\gamma)-A(\mu))_{a d}\right)$.
} 
In view of (14) we can re-express (5) in the form

$2 k_{\xi}-H^{(\xi)}=0, \quad 2 k_{\bar{\xi}}-H^{(\bar{\xi})}=0$.

Below, we will have to interpret these equations as canonical constraints à la Dirac rather than merely as equations determining the conformal factor; upon quantization, they will yield the Wheeler-DeWitt equation and the diffeomorphism constraint.

\section{Quantization}

To quantize the model, we replace the Poisson brackets (13) by commutators in the usual fashion:

$$
\begin{aligned}
& {[A(\gamma) \stackrel{\otimes}{ } \rightarrow(\mu)]} \\
& \quad=i \hbar[r(\gamma-\mu), A(\gamma) \otimes I+I \otimes A(\mu)] .
\end{aligned}
$$

The entries of the two-by-two matrix $A(\gamma)$ thus become operators acting on a Hilbert space to be specified below; note that on the l.h.s. of (16), we have a commutator of operators in Hilbert space whereas on the r.h.s. we have a commutator of ordinary matrices. This means in particular that the expansion (9) is no longer valid as an operator statement, but must be reinterpreted as a property of the states on which $A(\gamma)$ acts. The expressions for the Hamiltonians (14) remain unchanged. We write

$A(\gamma) \equiv \frac{i \hbar}{2}\left(\begin{array}{cc}h(\gamma) & 2 e(\gamma) \\ 2 f(\gamma) & -h(\gamma)\end{array}\right)$.

As is well known the Hamiltonians (14) can be diagonalized by means of the Bethe ansatz method [13]. For this purpose, one considers a lowest weight state $|p\rangle$ labeled by some analytic function $p=p(\gamma)$ subject to $\overline{p(\gamma)}=-p(-\bar{\gamma})$. This state is assumed to obey $h(\gamma)|p\rangle=p(\gamma)|p\rangle$ and $f(\gamma)|p\rangle=0$. The Bethe states are then

$\left|p ; v_{1}, \ldots, v_{M}\right\rangle:=e\left(v_{1}\right) \ldots e\left(v_{M}\right)|p\rangle$.

The parameters $v_{1}, \ldots, v_{M}$ must satisfy certain constraints called Bethe equations ( $M$ is the number of excitations) in order for the Hamiltonians (14) to act diagonally on (18). However, we will not need these constraint equations below, but we do make use of the Bethe states with unconstrained parameters (this is sometimes referred to as the "off-shell Bethe ansatz").
The off-diagonal operators $e(\gamma)$ and $f(\gamma)$ play the role of creation and annihilation operators, respectively (interchanging the role of $e(\gamma)$ and $f(\gamma)$ turns the representation "upside down"). The classical expansion (9) corresponds to the special choice

$p(\gamma)=\sum_{j=1}^{N} \frac{m_{j}}{\gamma-\gamma_{j}}$.

By fixing the number of poles in this fashion, we have restricted the full Hilbert space to its $N$ soliton sector, which can be represented as a direct product $\mathcal{H}^{(N)}=$ $\mathcal{H}_{1} \otimes \ldots \otimes \mathcal{H}_{N}$. Only on this subspace can we expand the operator $A(\gamma)$ in analogy with (9), with

$A_{j} \equiv \frac{i \hbar}{2}\left(\begin{array}{cc}h_{j} & 2 e_{j} \\ 2 f_{j} & -h_{j}\end{array}\right)$

where $h_{j}, e_{j}, f_{j}$ are the standard Chevalley generators of $\mathrm{SU}(2)$ obeying the commutation relations

$\left[h_{j}, e_{j}\right]=2 e_{j}, \quad\left[h_{j}, f_{j}\right]=-2 f_{j}, \quad\left[e_{j}, f_{j}\right]=h_{j}$

as a consequence of (16). By well known arguments, unitarity then requires each $\mathcal{H}_{j}$ to be a representation space of SU(2) with highest weight $m_{j} \in \mathbb{Z}$. We may therefore view the quantized $N$ soliton sector as a system of $N$ spins located at the points $(x, \rho)=$ $\left(w_{j}, 0\right)$ where the classical solutions of the system (5), (6) have singularities on the worldsheet [14]. Since the full Hilbert space is the direct sum of its $N$ soliton sectors, its structure resembles that of a Fock space with $N$ playing the role of a particle number operator (not to be confused with the number $M$ of excitations in (18)).

The central task is now to solve the quantum ana$\log$ of the constraints (15), which are nothing but linear combinations of the Wheeler-DeWitt equation and the diffeomorphism constraint for our model (corresponding to translations in $\rho$ and $x$, respectively), and read

$$
\left(2 k_{\xi}-H^{(\xi)}\right) \Phi=\left(2 k_{\bar{\xi}}-H^{(\bar{\xi})}\right) \Phi=0
$$

where $\Phi$ is the full quantum state (so-called "wave function of the universe"). Accordingly, we extend the phase space by the gravitational degrees of freedom $2 k_{\xi}, 2 k_{\bar{\xi}}$ and the variables $\xi, \bar{\xi}$. To proceed we must first construct an operator representation for these new 
phase space variables. From the canonical brackets given in [ 1$]$ we deduce the commutators

$$
\left[2 k_{\xi}, \xi\right]=\left[2 k_{\bar{\xi}}, \bar{\xi}\right]=i \hbar, \quad\left[2 k_{\xi}, \bar{\xi}\right]=\left[2 k_{\bar{\xi}}, \xi\right]=0 \text {. }
$$

We take

$2 k_{\xi}=i \hbar \frac{\partial}{\partial \xi}, \quad 2 k_{\bar{\xi}}=i \hbar \frac{\partial}{\partial \bar{\xi}}$.

The main advantage of this choice is that by representing $(\xi, \bar{\xi})$ as multiplication operators we salvage their interpretation as coordinates (otherwise the spectral parameter $\gamma$ would not remain a function but become a non-local differential operator and thus very awkward to deal with). It is then obvious that the two equations (21) are mutually compatible for the same reason that their classical counterparts (5) are. Recall that the worldsheet coordinates $(\xi, \bar{\xi})$ appear explicitly only because we have adopted the special gauge (4) identifying the dilaton field with one of the coordinates. In other words, this choice of gauge makes the quantum state $\Phi$ time-dependent through the identification of time with the "clock field" $\rho$. We note that this long suspected mechanism for the emergence of time from the "timeless" Wheeler-DeWitt equation here comes almost for free (see, e.g. [15] for a review and further references). In a covariant treatment the gauge choice (4) would have to be undone, and the full quantum state would be a functional of $\rho$ rather than a function of the worldsheet coordinates. The fact that through this choicc of gauge the field variables $2 k_{\xi}$ and $2 k_{\bar{\xi}}$, which originated from the conformal factor, become canonically conjugate to the worldsheet coordinates, is also in accord with the interpretation of $2 k_{\xi}$ and $2 k_{\bar{\xi}}$ as longitudinal target space momenta suggested in $[9,10]$, and can be construed as evidence for a "stringy" interpretation of the theory, with our treatment being analogous to the quantization of the string in the lightcone gauge.

Eqs. (21) now take the form

$i \hbar \frac{\partial \Phi}{\partial \xi}=H^{(\xi)} \Phi, \quad i \hbar \frac{\partial \Phi}{\partial \bar{\xi}}=H^{(\bar{\xi})} \Phi$

where in the $N$-soliton sector $\Phi$ is an $\mathcal{H}^{(N)}$-valued function of $(\xi, \bar{\xi})$. The explicit solutions of (22) may be obtained by exploiting a surprising link between (22) and the $\mathrm{KZ}$ equations [16] determining the
$N$-point correlation functions of the WZNW model, which read

$$
\frac{\partial \tilde{\Phi}}{\partial \gamma_{j}}=\frac{1}{\kappa} \sum_{k \neq j} \frac{\Omega_{j k}}{\gamma_{j}-\gamma_{k}} \tilde{\Phi}
$$

with an $\mathcal{H}^{(N)}$-valued function $\tilde{\Phi}$, where $\Omega_{j k}=\frac{1}{2} h_{j} \otimes$ $h_{k}+f_{j} \otimes e_{k}+e_{j} \otimes f_{k}(j \neq k)$ is a linear operator acting non-trivially only in $\mathcal{H}_{j}$ and $\mathcal{H}_{k}$. In [17] (see also [18-20]) it was shown that the general solution of (23) can be obtained in terms of the following integral representation over off-shell Bethe states

$\tilde{\Phi}=\int_{\Delta} W(\gamma, v)\left|p ; v_{1}, \ldots, v_{M}\right\rangle d v_{1} \ldots d v_{M}$

where

$$
\begin{aligned}
& W(\gamma, v)=\prod_{1 \leq k<j \leq N}\left(\gamma_{j}-\gamma_{k}\right)^{m_{j} m_{k} / 2 \kappa} \\
& \times \prod_{1 \leq s<r \leq M}\left(v_{r}-v_{s}\right)^{2 / \kappa} \prod_{r=1}^{M} \prod_{j=1}^{N}\left(\gamma_{j}-v_{r}\right)^{-m_{j} / \kappa}
\end{aligned}
$$

and $\Delta$ is a family of cycles in $v$-space which do not intersect the hyperplane where $W$ becomes singular; furthermore, $W$ should be single-valued on $\Delta$. For the precise definition of $\Delta$ and the proof of (24) see [17$19,21]$. It is important that the parameters $v_{r}$ in (24) are not subject to the Bethe equations. In [18] it was shown that the state (24) is annihilated by the constraint (12) iff

$M=\frac{1}{2} \sum_{j=1}^{N} m_{j}$.

Using (8) and (20) we can now verify that the $\mathrm{KZ}$ equations (23) imply that

$\Phi=\prod_{j=1}^{N}\left(\frac{\partial \gamma_{j}}{\partial w_{j}}\right)^{i \hbar_{j}^{m_{j}\left(m_{j}+1\right)}} \frac{\tilde{\Phi}}{4} \tilde{s}$

satisfies (22), provided the state $\Phi$ has vanishing "total spin" in accordance with (12) and we make the identification

$\kappa=-1 / i \hbar$. 


\section{Outlook}

To conclude we would like to briefly expose the extra complications that one faces when attempting to quantize dimensionally reduced gravity, whose distinct feature is the non-compactness of the configuration space inhabited by the propagating degrees of freedom. As we already mentioned, the relevant equations of motion can be formally obtained from our equations (5), (6) by replacing the compact SU(2) manifold by the non-compact coset space SU(1,1)/U(1), after which (6) becomes just the Ernst equation. Due to the division by the maximal compact subgroup, which is already necessary at the classical level in order to prevent the energy from being unbounded below, we now encounter new canonical constraints necessary to ensure the decoupling of unphysical negative norm states. In the case at hand, it turns out that, in fact, one must impose infinitely many such constraints corresponding to the maximal compact subgroup of the Geroch group $S \widehat{S(1,1)}$. Moreover, the representation spaces for each "spin" will be infinite-dimensional (unlike the spaces $\mathcal{H}_{j}$ above) because of the non-compactness of the group SU $(1,1)$.

\section{References}

[1] D. Korotkin and H. Nicolai, Phys. Rev. Lett. 74 (1995) 1272.

12] E. Witten, Nucl. Phys. B 311 (1988) 46;

S. Carlip, Lectures on $(2+1)$-dimensional gravity, preprint gr-qc/9503024
[3] B. de Wit, H.J. Matschull and H. Nicolai, Phys. Lett. B 318 (1993) 115.

[4] H. Kastrup and T. Thiemann, Nucl. Phys. B 425 ( 1994) 665.

[5] R. Graham, Phys. Rev, Lett. 67 (1991) 1381.

[6] K. Kuchar, Phys. Rev. D 4 (1971) 955.

[7] L. Faddeev and L. Takhtajan, Hamiltonian methods in the theory of solitons (Springer, Berlin, 1986);

V. Korepin, M. Bogoliubov and A. Izergin, Quantum inverse scattering method and correlation functions (Cambridge Univ. Press, Cambridge, 1993).

[8] L. Faddeev and N. Reshetikhin, Ann. Phys. 167 (1986) 227

[9] H. Nicolai, in: Recent aspect of quantum fields, Proceedings, Schladming 1991, ed. H. Mitter and H. Gausterer (Springer, Berlin, 1991).

[10] D. Korotkin and H. Nicolai, Nucl. Phys. B 429 (1994) 229.

[11] D. Maison, Phys. Rev. Lett. 41 (1978) 521 ; V. Belinskii and V. Zakharov, Sov. Phys. JETP 48 (1978) 985.

[12] M. Jimbo, T. Miwa, T. Mori and Y. Sato, Physica D 1 (1980) 80;

M. Jimbo, T. Miwa and K. Ueno, Physica D 2 (1981) 306.

[13] E. Sklyanin, J. Sov. Math. 47 (1989) 2473.

[14] D. Korotkin, Class. Quant. Grav. 100 (1993) 2587; Commun. Math. Phys. 137 (1991) 383.

[15] C.J. Isham, in: Canonical Relativity: Classical and Quantum, ed. J. Ehlers and H. Friedrich (Springer, Berlin, 1994).

[16] V. Knizhnik and A. Zamolodchikov, Nucl. Phys. B 247 (1984) 83.

[17] V.V. Schechtman and A.N. Varchenko, Lett. Math. Phys. 20 (1990) 279.

[18] H.M. Babujian J. Phys. A 26 (1993) 6981.

[19] N. Reshetikhin, Lett. Math. Phys. 26 (1992) 153.

[20] A.N. Varchenko, Asymptotic solutions to the KZ equations and Crystal Base, preprint hep-th/9403102.

[21] B. Feigin, E. Frenkel and N. Reshetikhin, Commun. Math. Phys. 166 (1994) 27. 